\section{LOS PETROGLIFOS COMO FORMA DE APROPIACIÓN DEL ESPACIO: ALGUNOS EJEMPLOS GALLEGOS}

\author{
GALICIAN ROCK ART AS A STRATEGY \\ OF APPROPRIATION OF SPACE
}

\author{
RICHARD BRADLEY $(*)$ \\ FELIPE CRIADO BOADO (**) \\ RAMÓN FÁBREGAS VALCARCE (***)
}

\section{RESUMEN}

Se presentan los primeros resultados de un proyecto de investigación en curso de realización cuyo objetivo es estudiar los petroglifos gallegos desde la perspectiva de la Arqueología del Paisaje. Se prima el análisis de las condiciones de emplazamiento de los grabados y de su relación con la topografía circundante, sobre el estudio estilístico y artístico de los motivos. Se consideran tres zonas distintas, dos de ellas situadas en diferentes puntos litorales y otra interior. Las observaciones realizadas permiten reconocer una relación significativa de los petrofligos con zonas de paso o con áreas en las que existen buenas reservas de pasto. Sin embargo, las diferencias entre las tres zonas permiten observar diferentes situaciones de presión sobre los recursos y, en relación con ello, diferencias notables en la distribución y densidad del arte rupestre de cada zona.

(*) Department of Archaeology. University of Reading. Whiteknights. PO Box 218. Reading RG6 2AA. Gran Bretaña.

(**) Departamento de Historia I. Facultad de Geografía e Historia. Universidad de Santiago de Compostela. 15703 Santiago de Compostela.

(***) Departamento de Historia, Arte y Geografía. Universidad de Vigo. Campus de Orense. As Lagoas. 32004 Orense.

El artículo fue remitido en su versión final el 13-IV-94.

\begin{abstract}
We offer in this paper the first results of a research project still in course, aiming at the study of Galician petroglyphs from the viewpoint of Landscape Archaeology. To that end, we emphasize the analysis of locational factors of carved rocks and their relationship with the surrounding landscape, rather than stylistic or artistic aspects. In this paper three main areas are considered, two coastal and another inland. Our observations point towards a significant link of the carved rocks with natural routes or good grazing areas. Nevertheless, the differences between the three zones considered would suggest situations of variable pressure on natural resources and, related to that, significant differences in the distribution and density of petroglyphs.
\end{abstract}

Palabras clave: Arqueología del Paisaje. Arte Rupestre. Petroglifos. Edad del Bronce. Galicia.

Key words: Landscape Archaeology. Rock Art. Petroglyphs. Bronze Age. Galicia.

\section{INTRODUCCIÓN}

El antropólogo M. Casimir (1992a, b), al estudiar la organización territorial de comunida- 
des itinerantes, sugiere que en zonas de ecología variada o en las que la densidad de población es elevada, los grupos sociales tienden a definir sus derechos territoriales de forma explícita. Coincidiendo con ello, la literatura etnográfica ha resaltado que el arte rupestre fue utilizado a menudo para delimitar recursos específicos (Hartley, 1992, cap. 4). Evidentemente ésta sería sólo una de las funciones del arte rupestre que, en general, parece haber sido el soporte de combinaciones complejas de valores sagrados y profanos (Layton, 1986). Por otra parte, tal y como Ingold (1986) ha mostrado, los grupos con un patrón de obtención de recursos itinerantes a menudo dejan "señales" para aquellos que utilizan el mismo medio. En este caso, los petroglifos son sólo un medio más por el cual se comunican los individuos de estos grupos entre sí.

En la Prehistoria se pueden documentar diferentes ejemplos de correlación entre movilidad y fenómenos arqueológicos que podrían haber servido para materializar esas reclamaciones territoriales (Álvarez, 1990), y, aunque en un orden distinto, en Galicia se verifica una estrecha relación entre líneas de tránsito a través del paisaje y el emplazamiento de los monumentos megalíticos (Infante et alii, 1992).

Independientemente de los ejemplos anteriores, las sugerencias de Casimir, Hartley e Ingold ofrecen una gran potencialidad para estudiar el arte rupestre prehistórico y, en nuestro caso, los petroglifos gallegos. Una de las razones fundamentales de su utilidad radica en el hecho de que para adoptar esa aproximación no es necesario comprender el significado original de las representaciones contenidas en el arte rupestre. Así, en nuestro caso, se observa por un lado que los grabados prehistóricos gallegos se concentran en zonas de topografía variada en las que existen importantes contrastes a lo largo del año en el crecimiento de la vegetación, precipitación, humedad y sequía. En momentos en los que el movimiento de animales parece haber sido significativo, sería lógico sospechar que, al menos en algunas de estas zonas, se darían situaciones de presión sobre los recursos. Por otra parte, parece existir, tal y como empezamos a estudiar en otro punto (Bradley et alii, 1994) una relación significativa entre la distribución de los petroglifos y las líneas de tránsito o movimiento a través del terreno. El presente trabajo pretende explorar y concretar ambas lí- neas de reflexión en relación con el arte rupestre gallego.

A menudo se ha argüido que el arte rupestre gallego es muy homogéneo. Sin embargo existen importantes diferencias en la distribución de los distintos tipos de motivos. Así, por ejemplo, mientras los motivos abstractos son comunes en la península de Morrazo, los grabados de animales son sobre todo abundantes en las tierras más al interior. Dentro de una investigación centrada en aspectos cronológicos, estilísticos y en las comparaciones a una escala geográfica amplia, estas diferencias no han sido analizadas en profundidad. En cambio, nosotros creemos que la especificidad de los petroglifos se puede y debe definir, en primera instancia, a una escala local, dejando para un momento ulterior la posibilidad de acceder a niveles más amplios, suprarregionales, de análisis. Por esta razón en el presente trabajo nos centraremos en el estudio de la relación entre los motivos grabados y su distribución en el paisaje.

$\mathrm{El}$ arte rupestre gallego es bastante simple. Los motivos son fundamentalmente de dos tipos: el primero es de carácter abstracto y se basa en cazoletas a las que se pueden añadir círculos concéntricos y líneas; el segundo lo constituyen las representaciones figurativas, entre las que destacan sobre todo los animales (ciervos y caballos) y, ocasionalmente, armas, antropomorfos y escenas de caza.

\section{METODOLOGÍA}

Si aceptamos la sugerencia planteada más arriba de que el arte rupestre puede jugar un papel activo en las estrategias de apropiación del espacio de grupos itinerantes, ¿cómo se puede comprobar en el campo esta propuesta? Nuestro trabajo se basa en tres premisas:

En primer lugar, si los petroglifos sirvieron como "mensajes" entre grupos y personas que no estaban presentes simultáneamente, entonces es obvio que los destinatarios de esos mensajes deberían ser capaces de localizar los petroglifos de algún modo. Esto implica presuponer la existencia de un código bien definido de emplazamiento y localización de los grabados y, por lo tanto, su existencia se puede verificar de algún modo comparando las características de emplazamiento de las rocas decoradas con las

T. P., 51, nº 2. 1994 
de las rocas no decoradas de las mismas zonas (Bradley et alii, 1993).

En segundo lugar, si los grabados formaron realmente parte de un sistema espacial más amplio, entonces deberíamos prever su aparición alrededor de recursos particulares. Del mismo modo que ha sido posible en Galicia definir de una forma bastante precisa el patrón de emplazamiento de los monumentos tumulares (Criado y Vaquero, 1993), podríamos esperar asimismo que los petroglifos mostraran una pauta igualmente previsible. Teniendo en cuenta las numerosas representaciones de animales, se podría esperar que existiera una estrecha relación entre la localización de los petroglifos y las zonas que fueran importantes para estos animales.

Por último, si el aserto de Casimir plantea que los grupos itinerantes marcan sus recursos sobre todo en zonas sujetas a competición, entonces deberíamos poder relacionar los petroglifos con situaciones de este tipo. De hecho ya hemos anotado que los grabados rupestres gallegos se localizan en zonas del país en las que, posiblemente, los grupos prehistóricos habrían necesitado utilizar estacionalmente diferentes tipos de entornos. En esas zonas existe un fuerte contraste entre las condiciones bioclimáticas de la costa y las de las tierras interiores. Dado que esas diferencias no son siempre de la misma magnitud, podríamos prever que las áreas que presentan un mayor contraste a lo largo del año habrían favorecido situaciones de mayor competencia (ver Bradley et alii, 1994: figs. 2 y 3). En estos casos, si los destinatarios del arte fueron muchos y muy diversos, entonces los petroglifos de esas zonas podrían haber contenido una mayor carga de información. Así, aunque en el presente sigamos sin poder leer sus mensajes, podríamos al menos esperar que en esas circunstancias las composiciones y los atributos formales de los grabados hayan sido más complejos que en las restantes áreas.

\section{TRABAJO DE CAMPO}

Para desarrollar este programa de investigación era básico seleccionar unas zonas de trabajo que poseyeran, por un lado, un arte rupestre representativo y, por otro, un contraste significativo entre costa e interior (Fig. 1). Así elegimos una zona litoral con un arte lo más

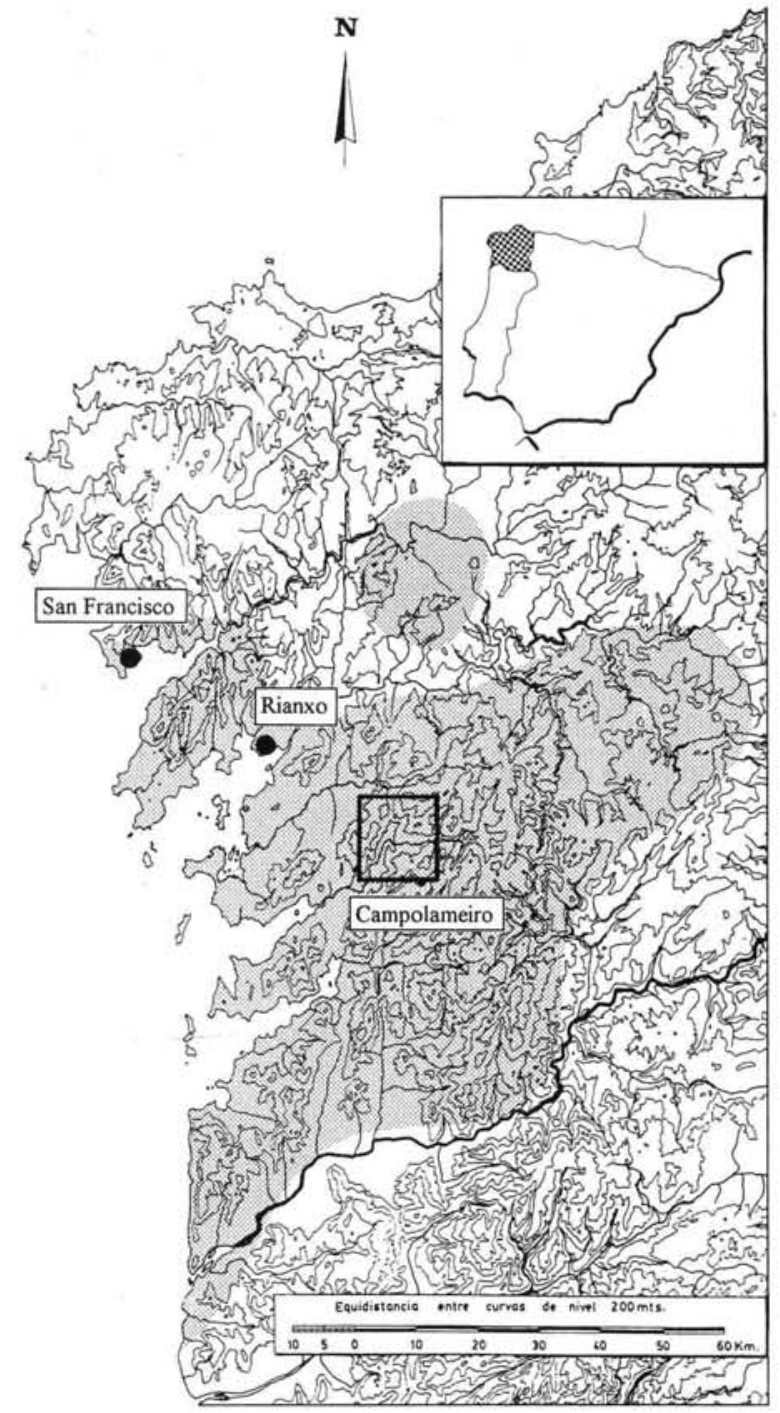

Fig. 1. Situación de las zonas de estudio.

simple posible: la costa norte de la ría de Muros presentaba petroglifos con motivos abstractos catalogados previamente (Eiroa y Rey, 1984) que representan bien el tipo de grabados característicos de la costa. Sin embargo, existen dos zonas litorales (las pequeñas penínsulas de Poio en la ría de Pontevedra (Aparicio, 1989: 85-160) y la de Rianxo en la de Arousa) excepcionales por poseer una gran cantidad de representaciones de animales. Por este motivo elegimos la segunda de ellas para completar nuestro muestreo en áreas costeras. Para ejemplificar las zonas interiores nos centramos en pequeñas áreas de Campo Lameiro (Álvarez, 1985-86, García y Peña, 1981). 
El trabajo de campo de Muros y Campo Lameiro se planteó de un modo similar. Se realizó una prospección intensiva para localizar todas las rocas con petroglifos, se compararon las características de las rocas grabadas con las no grabadas y, además se estudió la situación de los petroglifos en relación con la microtopografía y su disposición sobre la roca. En Rianxo (área estudiada recientemente en Bonilla, 1993), no necesitamos realizar un trabajo de campo tan intensivo y nos limitamos a caracterizar la distribución y localización de los petroglifos.

\section{RESULTADOS}

De un modo muy esquemático, presentaremos las características topográficas y las pautas de emplazamiento de los petroglifos reconocidos en cada uno de los casos de estudio.

\section{San Francisco, Muros}

Esta zona está conformada por tres ambientes bien diferenciados e interrelacionados entre sí: (a) un pequeño valle litoral, fértil y ocupado por las aldeas y tierras de cultivo intensivo tradicionales, y en cuyo centro se sitúa el Monasterio de San Francisco; (b) un valle estrecho y accidentado que une el área anterior con las tierras superiores y que se podría definir casi como un "desfiladero»; y (c) una cuenca elevada, rodeada por cumbres más altas y accidentadas, y destinada tradicionalmente a aprovechamientos extensivos (pasto de verano y cultivo de roza). La distribución de los petroglifos y sus motivos en relación con estas diferencias ambientales es muy significativa (Fig. 2).

Los límites de la cuenca inferior están marcados por tres petroglifos con representaciones de escasa complejidad (círculos concéntricos con líneas radiales) situados en zonas desde las que dominan amplias panorámicas sobre la zona litoral y que marcan, de hecho, el borde de las tierras abrigadas inferiores adecuadas para su ocupación continuada. Por encima de estos petroglifos aparecen algunos grabados con representaciones sencillas (meras cazoletas), situados en zonas accidentadas y prominentes, que dominan los petroglifos anteriores y la cuenca inferior en su totalidad; el más septentrional de ellos domina el trazado del desfiladero en el que, en cambio y al igual que en los restantes valles que conducen a la cuenca superior, no han aparecido petroglifos.

En la cuenca superior aparece, sobre una roca de grandes proporciones, el petroglifo bien conocido de Laxe das Rodas, que posee las representaciones más complejas de todo el sistema. Se sitúa casi en la entrada sur de la cuenca, al lado de la línea de tránsito que atraviesa ésta y en el punto en el que convergen los dos desfiladeros que, desde la zona litoral, conducen a ella. Su posición resulta visible desde cualquier punto de esta cuenca. Sin embargo no lo es desde las tierras bajas y litorales que, además, tampoco se divisan desde el petroglifo. Los motivos representados sólo se pueden observar desde la parte más elevada de la roca y mirando en dirección hacia el interior de la cuenca. Al igual que en uno de los petroglifos del valle inferior, en la parte más alta de la roca se encuentran varias cazoletas.

Alrededor de Laxe das Rodas se han descubierto siete rocas grabadas con cazoletas (en algún caso presentan hasta cinco cazoletas juntas y en otros aparece sólo una). Se localizan todas sobre rocas prominentes, en la parte más elevada de la cuenca y bordeándola. Cuatro de ellas se encuentran en zonas de entrada a dicha cuenca: dos ( 1 y 3 ) dominan los dos valles o desfiladeros que unen a ésta con las tierras litorales, una (5) se sitúa en un punto desde el que es intervisible con los petroglifos inferiores y la última (4) domina el punto donde la cuenca converge con un amplio valle que desciende hacia el norte (Fig. 2).

En síntesis nos encontramos delante de un conjunto aparentemente muy sistemático, en el que los puntos importantes de la topografía (la cuenca inferior y la superior) son resaltados por representaciones complejas que, a su vez, son dominadas por meras cazoletas. Estas últimas se emplazan sobre posiciones conspicuas y prominentes, cerca de hecho de su cota superior, lo que sugiere que fueron realizadas en lugares cuya significación ya estaba bien establecida con anterioridad. Esta distribución, por lo tanto, no parece fruto del azar, sino que se sitúa en relación con dos zonas bien definidas cuya utilización, teniendo en cuenta que una es abrigada y apta para su ocupación continuada y otra más alta y adecuada para su uso extensivo, podría haber sido complementaria. 

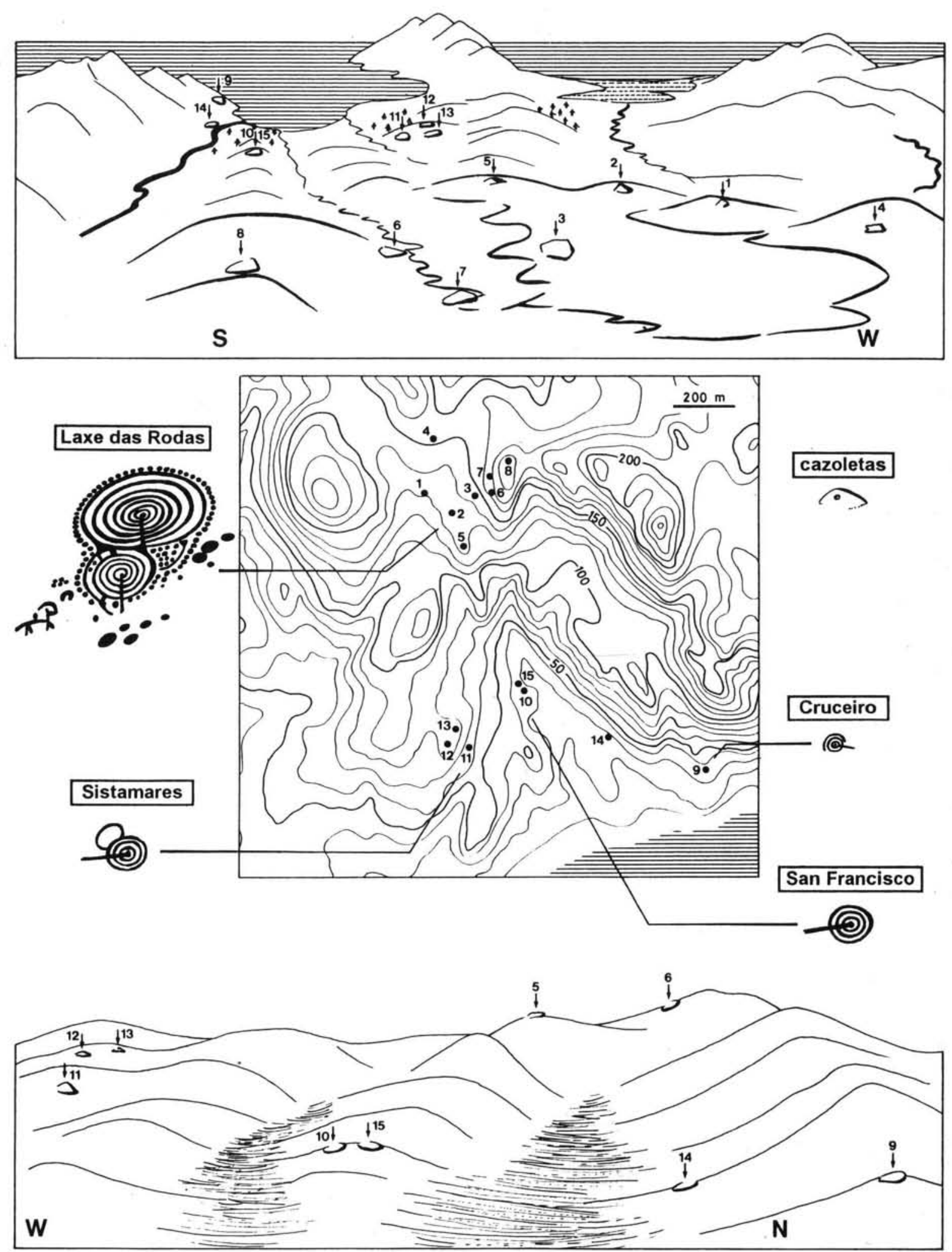

Fig. 2. Distribución y emplazamiento de los petroglifos en la zona de San Francisco. Los petroglifos con cazoletas se representan con un punto negro. Las vistas panorámicas dan un detalle de los niveles superior e inferior de la cuenca topográfica y de la situación relativa de los petroglifos en relación con ellos. 


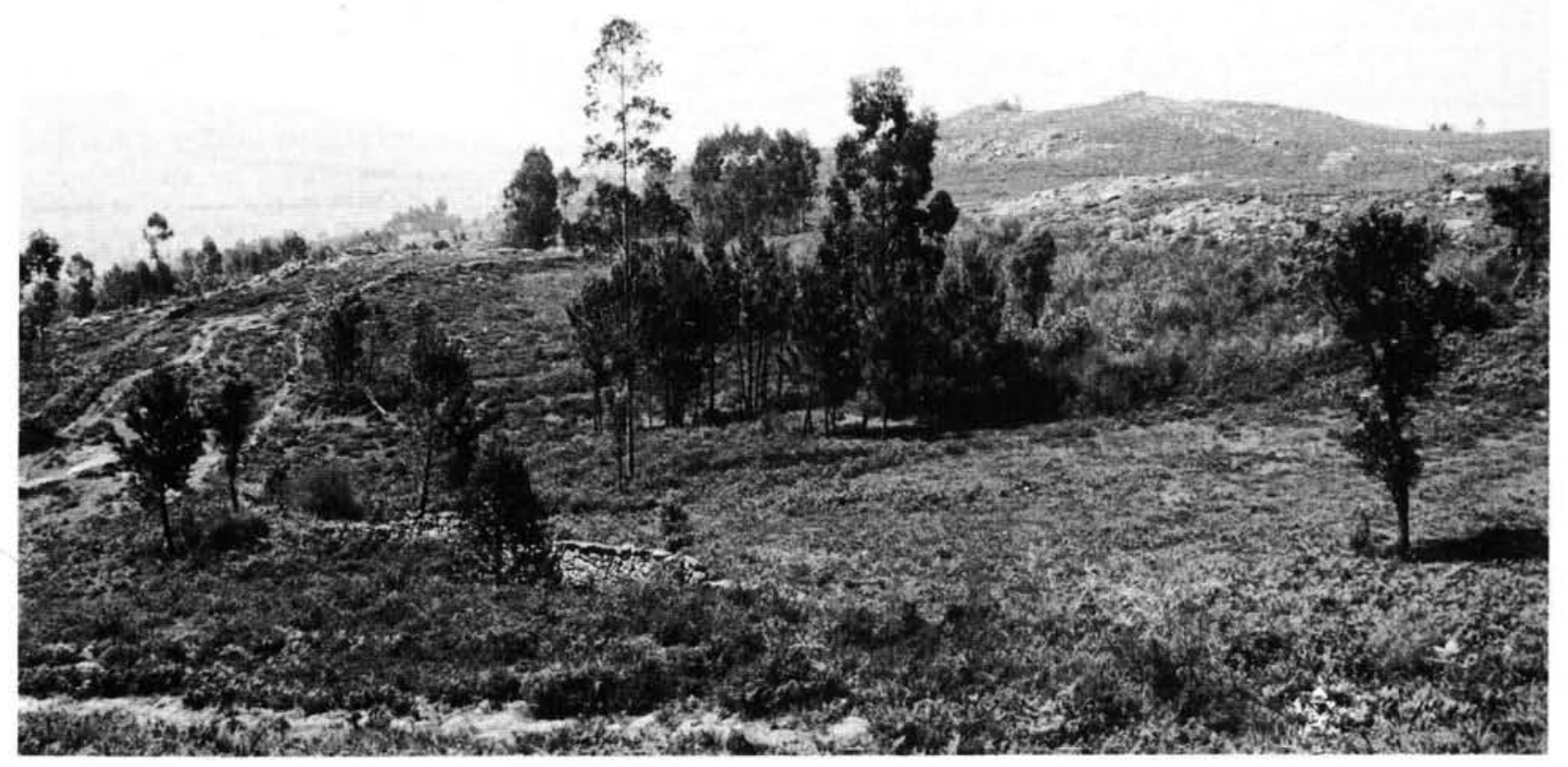

Lám. I. Cuenca de Chan da Lagoa, en Campo Lameiro.

\section{Campo Lameiro}

Esta zona, capital en el arte rupestre gallego y que marca el centro aproximado de su distribución, contrasta con la anterior en que en ella, además de motivos abstractos, son frecuentes las representaciones de animales. En ella se han analizado en detalle tres grupos principales de grabados (Paredes, Fentáns y Chan da Lagoa) y considerado datos procedentes de otros dos (Caneda y Rotea de Mendo) (Bradley et alii, 1994).

La topografía, condiciones naturales y uso del suelo en esta zona son muy distintas a las de la anterior. Los petroglifos se encuentran sobre todo en áreas de monte (1), en las partes elevadas de los valles y en las tierras altas. Si hubieran existido en las zonas bajas en torno al Lé-

(1) En Galicia se designa con esta categoría a las zonas dedicadas a usos extensivos, que generalmente conjugaban pasto extensivo, obtención de tojo para abonado y cultivo de rozas.

T. P., $51, \mathrm{n}^{\circ} 2.1994$ rez, la utilización intensiva de las mismas habría hecho muy difícil su conservación hasta la actualidad. Los grabados se sitúan en torno a pequeñas cuencas cerradas, ocupadas por una braña o turbera incipiente, y que son espacios de reserva de humedad y pasto durante la estación seca, especialmente pronunciada en esta zona de Galicia.

Los petroglifos se sitúan dominando generalmente esas cuencas y dando en cambio la espalda a las panorámicas amplias sobre valles y tierras bajas (Lám. I). Del mismo modo, aunque se localizan a menudo en posiciones conspicuas, existen en sus inmediaciones rocas que poseen emplazamientos más prominentes. Además, se sitúan a lo largo de las líneas de tránsito y caminos que atraviesan estas zonas y discurren alrededor de sus cubetas interiores. En los grabados son frecuentes las figuraciones de caballos y ciervos. Éstos raramente se representan aislados, sino en grupos, de perfil y orientados casi siempre en la misma dirección que, sorprenden- 


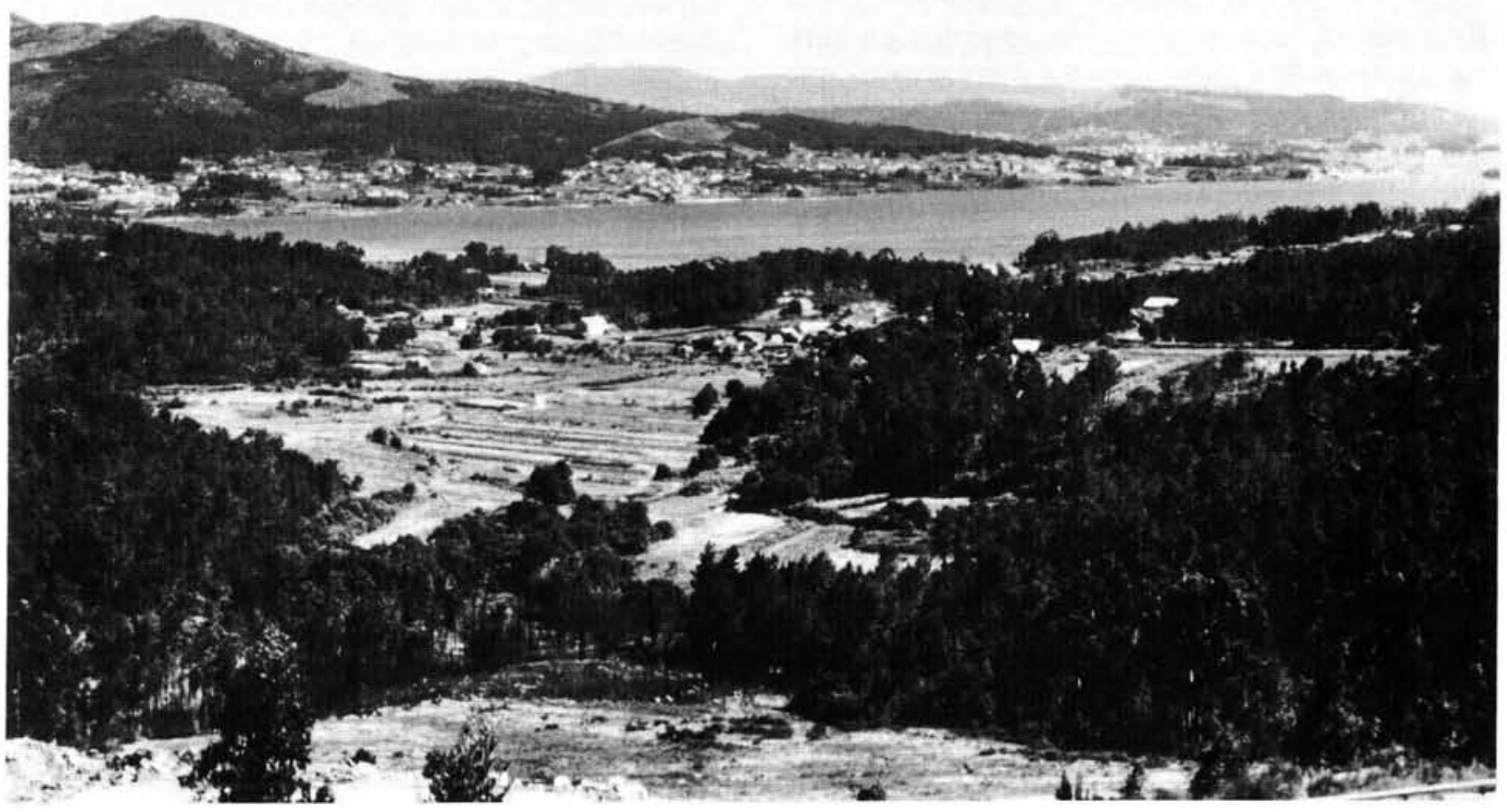

Lám. II. Panorámica de la zona estudiada en Rianxo desde el petroglifo con armas de Foxa da Vella.

temente, coincide casi siempre con la de la línea de tránsito a la que se asocia el petroglifo.

Cuando los petroglifos aparecen en otras posiciones, fuera de las cuencas anteriores, se localizan a lo largo de los caminos que conducen a fuentes o que comunican cuencas individuales entre sí. Excepcionalmente algunos se emplazan fuera de los límites de valles estrechos y cuencas tratados hasta aquí, aislados en relación con los sistemas de grabados asociados a éstas y sobre rocas visibles que controlan amplias panorámicas visuales. En estos casos aparecen grabados de ciervos con grandes cornamentas que parecen representar machos adultos durante la estación de celo, que es cuando estos animales ocupan las partes altas del terreno y adoptan poses agresivas. Con todo, no debemos pensar que estos ejemplos ofrezcan una representación literal de la fauna local, sino que, al igual que ocurre con las figuraciones de armas que poseen el mismo tipo de localización, parecen denotar un sentido de agresión competitiva.

\section{Rianxo}

Aquí los petroglifos se extienden entre la costa y una penillanura situada $2 \mathrm{~km}$. hacia el interior, con diferencias de cota entre 20 y 110 m. (Lám. II). La modificación del paisaje introducida por el desarrollo de la zona hace que en la actualidad se diferencien dos áreas de concentración de petroglifos: la más baja se distribuye en torno a valles litorales estrechos, y la más elevada se concentra en la citada llanura. Más allá de ambas áreas aparecen petroglifos aislados. Mientras en las dos primeras zonas son frecuentes lo grabados de animales, fuera de ellas el arte es más abstracto, semejante al de San Francisco (2).

(2) La única excepción en este sentido es el petroglifo de "A Foxa da Vella” (Calo y González, 1980), en el que se combinan representaciones de motivos circulares y armas y que se sitúa tierra adentro, en una zona elevada y dominando una vasta panorámica. 
Aunque la distorsión que las labores de extracción de piedra han ocasionado en la distribución de los petroglifos no permite realizar análisis detallados, se observa que la localización de los petroglifos de esta zona responde a un patrón muy consistente. Al igual que los grabados de Campo Lameiro, dominan una serie de valles o cuencas abrigadas, aunque en este caso esos valles unen la costa y las tierras más interiores y elevadas. Los animales se representan moviéndose a lo largo de esa línea y parece que sólo son característicos de las dos concentraciones principales, mientras que el arte abstracto posee una posición más periférica. Dentro de esas dos concentraciones se perciben algunos rasgos de variación interna aunque son de carácter incierto.

\section{CONSECUENCIAS}

Las tres zonas que hemos revisado hasta aquí presentan rasgos comunes y distintivos. Cada una conforma un sistema de arte rupestre específico. A continuación intentaremos correlacionar esos sistemas entre sí.

El sistema más simple es el de San Francisco. La distribución de petroglifos no es muy densa, se localizan sobre afloraciones prominentes y conspicuas y la variedad de motivos es pequeña. La utilización de círculos concéntricos y cazoletas fue suficiente para delimitar las áreas más productivas del entorno: un pequeño valle abrigado al borde de la ría y una cuenca elevada de terrenos aptos para usos extensivos. La distribución de los motivos sobre las rocas refleja la distribución de los petroglifos en el paisaje: los motivos circulares ocupan las zonas más deprimidas de las afloraciones, en tanto las cazoletas se sitúan en sus puntos prominentes (3).

En las otras dos zonas, en cambio, el arte es más complejo y su distribución más densa. En ambas existen representaciones de animales, pero en Rianxo éstas se concentran en grupos compactos que están rodeados por motivos abstractos en las zonas vecinas. Al igual que en San Francisco, los petroglifos se concentran en pe-

(3) Aunque este dato nos llevaría a cuestiones distintas, conviene decir que los únicos ejemplos de petroglifos con representaciones naturalistas existentes en esta zona geográfica, ocupan una posición topográfica totalmente distinta a las dos que documentamos en San Francisco, tal es el caso del petroglifo de Cova da Bruxa (Eiroa y Rey, 1984: 62 y ss.). queños valles litorales que se extienden hacia el interior y se sitúan sobre afloraciones prominentes descartando, en cambio, rocas menos visibles. Y a diferencia de San Francisco, los petroglifos no dominan amplias panorámicas, sino que parecen haberse situado para controlar áreas concretas del entorno. La orientación de los animales representados coincide con la línea topográfica entre el litoral y las zonas altas interiores.

Campo Lameiro comparte muchos de los rasgos anteriores, aunque el patrón de emplazamiento de los petroglifos y la variedad de motivos representados son más complejos. La densidad de petroglifos es muy elevada pero éstos se distribuyen a lo largo de una superficie mucho más amplia. Al igual que en Rianxo, los petroglifos están relacionados con el control visual de zonas concretas, generalmente brañas y cuencas estrechas que constituyen áreas de reserva de pasto para el verano. Sin embargo los petroglifos evitan situarse sobre las afloraciones más conspicuas y se sitúan a lo largo de las sendas seguidas en sus movimientos por los animales salvajes. Los animales representados se orientan en el mismo sentido que esas sendas y, en ocasiones, flanquean a los motivos circulares, configurando un patrón que replica en cierta medida la distribución genérica de los petroglifos sobre el entorno (rasgo que de algún modo aparece también en San Francisco). Sólo los petroglifos con armas o machos de grandes cornamentas buscan emplazamientos más prominentes y panorámicas más amplias (rasgo que también está presente en Rianxo -ver lámina II).

En las tres zonas que hemos analizado existe una estrecha relación entre el emplazamiento de los petroglifos y la localización de los recursos críticos del entorno, representados fundamentalmente por la presencia de brañas y cuencas húmedas y equiparable en cierta medida a la relación significativa percibida por F. Méndez (1991) entre este mismo tipo de áreas y yacimientos habitacionales de la Edad del Bronce. En dos de ellas (Rianxo y Campo Lameiro) también se documenta una clara relación entre los petroglifos y las líneas de tránsito (4). En síntesis, se puede argüir que el emplazamiento y localización de las representaciones se ajusta a un patrón homogéneo y predecible que, ade-

(4) En San Francisco únicamente Laxe das Rodas presenta una relación de este tipo. 
más, podría haber servido para definir el acceso a recursos específicos dentro de un patrón de subsistencia de carácter itinerante, al menos en ciertos aspectos. Cuando menos, existen evidencias suficientes para mantener esta idea como hipótesis de trabajo (5).

Por otra parte, tanto en la densidad de los petroglifos como en su variedad de motivos, cada una de las tres zonas consideradas presenta un distinto nivel de complejidad. Si aceptamos la sugerencia de M. Casimir (1992a) presentada al principio de este trabajo, y aceptamos asimismo que los petroglifos galaicos actuaron como un sistema de apropiación del espacio, entonces debemos sospechar que, en aquellas zonas que hubieran sufrido una presión mayor sobre los recursos, el arte rupestre habría poseído un mayor grado de complejidad.

Contrastes de este tipo (en precipitación, sequía, temperatura, humedad y amplitud de período vegetativo) se encuentran al comparar la zona interior de Campo Lameiro con las áreas de costa (Bradley et alii, 1994: figs. 2 y 3). Pero esta misma escala de contrastes se documenta entre el litoral de la ría de Arousa y el interior de la península de Barbanza, situándose, además, su gradiente máximo en la península de Rianxo. Esta circunstancia hace de esta zona una región óptima para la migración de cérvidos pero, sobre todo, significa que en ella los recursos habrían tenido una distribución estacional. En San Francisco y en otras zonas del litoral gallego con petroglifos no se documenta en cambio un contraste de este tipo. Podría ser ésta la razón de que en Rianxo la distribución y variedad de los petroglifos presente un carácter tan peculiar.

Sin embargo, esta última interpretación deberá ser definida a la luz de ulteriores investigaciones. A fin de cuentas, el propósito de este breve trabajo es meramente apuntar la potencialidad de una metodología basada en la Arqueología del Paisaje para el estudio del arte rupestre galaico y, simultáneamente, presentar los primeros datos relativos al análisis de los petroglifos en su dimensión espacial. Esto, evidentemente, no agota todos los niveles significati-

(5) La relación entre zonas de reserva de pasto y yacimientos de la Edad del Bronce ha sido definida por Fidel Méndez como un elemento indicativo de un patrón de subsistencia en el que el ganado vacuno y la movilidad estacional habrían jugado un papel social determinado; véase su trabajo en el número anterior de este volumen. vos distintos en los que el análisis del arte rupestre debe ser realizado.

\section{Agradecimientos}

Este artículo forma parte de un proyecto sobre el arte gallego actualmente en curso con la autorización de la Dirección Xeral do Patrimonio Histórico e Documental de la Xunta de Galicia y la financiación parcial de esta misma institución, la British Academy, la Reading University y la Universidade de Santiago de Compostela. La labor de documentación previa fue realizada por J. Amado y D. Soto, y los dibujos delineados por Anxo Rodríguez Paz. Además, los autores queremos agradecer a las siguientes personas su colaboración y ayuda en diferentes aspectos del trabajo: Andrés Bonilla, Ángel Concheiro, Dolores Gil, Matilde González, Fausto Infante, Fidel Méndez, Steve Mithen, Antonio de la Peña, Rafael Penedo, Sofía Quiroga, Pepa Rey, Manuel Santos y Maruchi Tallón.

\section{BIBLIOGRAFÍA}

Álvarez Núñez, A. (1985-86): «Los petroglifos de Fentáns (Cotobade)». Pontevedra Arqueológica, 2: 77-125.

Álvarez SANChis, J. (1990): «Los verracos del valle del Amblés: del análisis espacial a la interpretación socioeconómica». Trabajos de Prehistoria, 47: 201-233.

Aparicio CASADO, B. (1989): «Arqueología y Antropología Cultural de la margen derecha de la Ría de Pontevedra». Diputación Provincial de Pontevedra.

Bonilla Rodriguez, A. (1993): «Inventario de grabados rupestres del Ayuntamiento de Rianxo». Consellería de Cultura. Dirección Xeral do Patrimonio Histórico e Documental (memoria inédita). Santiago de Compostela.

Bradley, R., Harding, J., Mathews, M. y Rippon, S. (1993): «A field method for analysing the distribution of rock art». Oxford Journal of Archaeology, 12: 29-43.

Bradley, R., Criado Boado, F. y Fábregas Valcarce, R. (1994): «Rock art research as landscape archaeology. A pilot study in Galicia, north-west Spain». World Archaeology, 25, 3: 374-390.

CAlo Lourido, F. y González Reboredo, J. (1980): «Estación de arte rupestre de Leirom. Gallaecia, 6: 207216.

CASIMIR, M. (1992)a: «The dimensions of territoriality: an introduction». En M. Casimir y A. Rao (eds.): «Mobility and Territoriality». Berg. New York: 1-26. 
- (1992)b: «The determinants of rights to pasture: territorial organisation and ecological constraints». Mobility and Territoriality. Berg. New York: 153-203.

EiroA, J. y REY, P. (1984): "Guía de los petroglifos de Muros». Concello de Muros. Santiago de Compostela.

García Alen, A. y Peña Santos, A. (1979): "Grabados Rupestres en la Provincia de Pontevedra». Fundación P. Barrié de la Maza. Coruña.

HARTLey, R. (1992): «Rock Art on the Northern Colorado Plateau". Aldershot. Avebury.

Infante Roura, F., Vaquero lastres, J. y Criado BoADO, F. (1992): «Vacas, caballos, abrigos y túmulos: definición de una geografía del movimiento para el estudio arqueológico". Cuadernos de Estudios Gallegos, XL (105): 21-39.
INGOLD, T. (1986): «Territoriality and tenure: the appropriation of space in hunting and gathering societies». En T. Ingold (ed.): «The Appropriation of Nature», Manchester University Press. Manchester: 130-64.

LAYTON, R. (1986): «Political and territorial structures among hunter-gatherers». Man, 21: 18-33.

MÉNDEZ FERNÁNDEZ, F. (1991): «El campaniforme tardío: entre un pasado monumental y una cerámica conspicua». En F. Criado Boado, A. Bonilla Rodríguez, D. Cerqueiro Landín, M. Díaz Vázquez, M. González Méndez, F. Infante Roura, R. Penedo Romero, E. Rodríguez Puentes y J. Vaquero Lastres, «Arqueologia del Paisaje. El área Bocelo-Furelos entre los tiempos paleolíticos y medievales». Consellería de Cultura, $\mathrm{Ar}$ queoloxía-Investigación, Santiago de Compostela, 6: 173-197. 